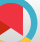

\title{
Perceived Advantages and Disadvantages of Sex Education in Young Women: A Qualitative Study
}

\author{
Azam Rahmani, ${ }^{1}$ Effat Merghati-Khoei, ${ }^{2,}{ }^{,}$and Arezoo Fallahi ${ }^{3}$ \\ ${ }^{1}$ Nursing and Midwifery Care Research Center, Tehran University of Medical Sciences, Tehran, IR Iran \\ ${ }^{2}$ Iranian National Center of Addiction Studies (INCAS), the Risk Behaviour Institution, Tehran University of Medical Sciences, Tehran, IR Iran \\ ${ }^{3}$ Environmental Health Research Center, Kurdistan University of Medical Sciences, Sanandaj, IR Iran \\ "Corresponding author: Effat Merghati-Khoei, Iranian National Center of Addiction Studies (INCAS); the Risk Behaviour Institution, Tehran University of Medical Sciences, \\ Tehran, Iran. Tel/Fax: 09132133513, E-mail: effat_mer@yahoo.com
}

Received 2016 January 31; Revised 2017 July 01; Accepted 2017 November 12.

\begin{abstract}
Background: Although the importance of sex education for the youth is well documented in the literature around the world, sex education remains controversial in Iran.

Objectives: The aim of this study was to explore Iranian women's beliefs and perceptions regarding advantages and disadvantages of sex education.

Patients and Methods: In this qualitative inquiry, we interviewed 41 young women aged 18 - 35 . Focus group discussions and individual interviews were used to collect the data. Conventional content analysis was used for data analysis. Multiple data collection methods, maximum variation sampling, and peer checks were applied to empower the rigor of study.

Results: A total of 5 themes were categorized into 2 main domains: advantages and disadvantage of sex education. The first domain was conceptualized by increased knowledge, normalization of sexual and reproductive issues, and self-efficacy; the later domain by: motives for initiating sexual behavior and tainted modesty.

Conclusions: Since most young women believed that sex education is beneficial for them, an effective, appropriate, and culturallyapproved program could be designed for sex education.
\end{abstract}

Keywords: Sex Education, Advantage, Disadvantage

\section{Background}

The importance of sex education for young people is well documented (1). According to the united nations population fund (UNFPA), sex education could influence young people's attitudes, beliefs, interactions, and intimate relationships (2). Worldwide, people experience sexual maturity earlier and marry at later ages; thus, they are more probable to engage in premarital sexual behavior. Furthermore, adolescents and youth are at greater risk for sexually transmitted infections; therefore sex education for this group is essential (3). Sex education remains controversial in Iran due to the fact that any extra marital relationship is legally, culturally, and religiously forbidden (4). Also, many people with specific moral views are worried about becoming sexually active as a consequence of sex education in adolescents (5).

We believe sex education is more critical in the Iranian society than others due to some rationales. About $20 \%$ of the Iranian population are made by youth (6); these young individuals are interested in postponing their marriage; the unique institute that permits them to engage in sexual behaviors. However, despite the law, they begin their sexual activities before marriage $(4,7)$. According to a study, $27.7 \%$ of Iranian youth experienced their first coition at age 15 and $68.2 \%$ of sexually active youth engaged in at least 1 sexual risk-taking behaviors, (8). Also, risky sexual behavior, such as unprotected sexual intercourse and multiple sexual partnerships, is the second main route of HIV transmission in Iran (9). Alarmingly, HIV infections in Iran are rising and risky sexual behaviors is a cause for a growing percentage of these infections (10).

However, sex education may not simply be plugged into the curriculum. Therefore, to design a culturally appropriate, comprehensive, and effective sex education, attitudes and concerns of youths, parents, and the society as a whole need to be considered (11). Women's perceptions regarding sex education could be applied for making a culturally appropriate policy for better decision making regarding the integration of sex education into the curricu- 
lum.

Globally, there have been many previous studies focusing on sex education in universities, schools, and high-risk groups $(1,12,13)$, however, few studies have been done regarding sex education in Iran.

\section{Objectives}

The aim of this paper is to explore Iranian women's beliefs and perceptions about advantages and disadvantages of sex education.

\section{Patients and Methods}

This was a qualitative study. Few sexual data were available in the Iranian society; therefore, we selected conventional content analysis (14). Young women $(n=41)$ aged 18 -34 were recruited.

In the first step, focus group discussions were applied. Although Iran is a Muslim society, new generations may not think that talking about sexuality matters could be against religious beliefs (15). In FGDs, we purposefully approached all young women irrespective of their sexual experiences and receiving/not receiving sex education. The FGDs were facilitated by defining sex education. In addition, a semi-structured inventory was used, which began with open-ended question: 'have you ever received any education related to sexuality?'. Afterwards, subsequent questions were asked based on the responses that participants provided.

In the FGDs, women who engaged in sexual activity were not eager to openly talk about their sexual experience, however, we could identify them while they discussed their beliefs; for example, several young women believed engaging in premarital sexual behaviors is a natural phenomenon and each woman, like a man, could enjoy it.

Maximum variation sampling (different socioeconomic statuses and age groups, high and low religiosity, and sexual experience types) was used for greater transferability of data (16). Participants had various educational statuses. In total, we held 5 focus group discussions and 10 individual interviews. After 4 FGDs and 8 interviews, data saturation was achieved.

Qualitative content analysis as well as Graneheim and Lundman's approach was applied for data analysis. Data analysis started while the data were collected. Every interview and FGD was transcribed verbatim and analyzed before the next interview and FGD.

There was an expert second coder in the study. A total of 5 participants of the study were asked to review the summary of the interviews (member check). We checked substantive codes and themes with 4 people who were experts in analyzing the qualitative data (peer check) (16). Data collection with multiple methods (individual interview and focus group discussion) enhanced credibility and dependability of data (16).

This study was approved by the ethics committee of the school of medicine of the Tarbiat Modares university. We dedicated a pseudonym to the participants and informed them that taking part in the research was voluntary, their confidentiality would be preserved, and no name would be identified in the study publications. We obtained informed written consent from all young women.

\section{Results}

Four main themes emerged in the data analysis process: effects of sex education, principles of sex education, content of sex education, and organizations related to sex education. In this paper, the main theme that has been presented is 'effects of sex education'. Descriptions of other themes will be reported in subsequent manuscripts. The subthemes of 'effects of sex education' included: a, increased knowledge; b, normalization of sexual and reproductive issues; c, self-efficacy as advantages of sex education; $\mathrm{d}$, tainted modesty, and e, Motive for initiating sexual behavior as disadvantages of sex education.

\subsection{Advantages of Sex Education}

Three themes emerged from the analysis as advantages of sex education: a, increased knowledge, b, normalization of sexual and reproductive issues, and c, self-efficacy.

\subsubsection{Increased Knowledge}

More than 2/3 of the participants believed that sex education could increase their knowledge and promote their understanding of sexual and reproductive health. Shabnam (29 years) (These names are pseudonyms) who received sex education stated that:

'... I may have a sexual encounter and in this case, having sexual knowledge is better than being uninformed ... having sexual knowledge provides an appropriate view regarding all aspects of such a sensitive issue.' (FGD1)

A number of participants stated that they have been curious regarding sexuality-related knowledge. Zohreh (25 years old) who received sex education highlighted that:

'During my adolescence, I was very curious about the relationship with my boyfriend. Sex education helped me have a better understanding about intimate and physical relationships.'(ind.inw)

Participants believed that sex education is an important means to gain knowledge, particularly compared to learning from parents or peers. Most participants (more 
than 90\%) stated that they have learned sexuality from their peers while they believed that peers were not the right people to learn from. Furthermore, most participants believed that parents were not able to understand their sexual needs and therefore were not able to educate them or answer their sexuality-related questions.

\subsubsection{Normalization of Sexual and Reproductive Issues}

Approximately all participants reported there is a strong sensitivity around sexuality matters in Iran, as they could not speak, study, or search about sexuality. They regarded sex education as a means to overcome the disapprovals surrounding sexual matters. They believed that if sex education was provided, they would be able to accept sexuality as a natural phenomenon. Mandana (26 years) who had received sex education stated:

'I remember that when one of my classmates had experienced her menstruation for the first time, it was very embarrassing for her. Then, one of our teachers explained it as a natural event. Afterward, the sensitivity of the topic was gone and talking about menstruation became normal for us.' (ind.inw)

A devoted religious student (24 years) who has not received sex education stated:

'You know, it [sex education] will help people to think that sexuality is natural and it is necessary for everyone... it [sexuality] should be accepted as a reality' (FGD3)

Also Fatemeh (29 years) who has not received sex education reported her experience:

'Before I entered the university, I believed that anything related to sexuality is dirty. After I was accepted to the university, I accidently found a book regarding sexual and reproductive health. The content of that book was very interesting for me. In fact, before reading that book, I was avoiding accepting sexual issues, but after getting information, I felt God has created sexual issues as a natural complementary aspect of human beings.'(ind.inw)

Overall, participants believed that if sex education was provided, the sensitivity surrounding sexuality would decline and people could speak openly about it.

\subsubsection{Self-Efficacy}

Most participants stated that sex education could protect them from risky sexual behaviors. They found themselves at a potential risk during their sexual activity because they lacked a sense of control to reject the sexual act or use a condom. Shirin (23 years), a single and sexually active girl, stated:

'One year after our friendship, my boyfriend wanted me to have anal sex. I loved him and was not able to reject his request. At the same time as our relationship, our school provided some information about sexually transmitted infections. Afterwards, I was informed that unprotected anal sex was a risky sexual act and therefore I decided not to let him have anal sex with no protection.' (ind.inw)

One of the important pathways for practicing safe sex is building self-efficacy. The importance of safe sex and condom use were pointed out by the participants; Mahasti, 24 years old, who has received sex education stated:

'In my opinion, it is not time to say sexuality is dirty! It is better to say how to use the condom or suggest it to your partner. When I wanted to have sex with my first boyfriend, it was difficult for me to suggest using the condom to him. I thought if I suggest using condoms, he would think I have doubt about his health or I have a sexually transmitted infection; then, I preferred not to say anything about it.' (ind.inw)

Participants explained that sex education might provide a situation for them to think about their sexual relationships before they have sexual encounters; therefore, they could make the best decision in this situation.

\subsection{Disadvantages of Sex Education \\ 4.2.1. Motive for Initiating Sexual Behavior}

Some participants believed that sex education could operate as a sexual stimulus. A religious participant, Razieh (31 years), who has not received sex education, seemed to express this conservative objection well:

'With providing sex education, books and classes will become a channel for people's sexual gratification. Sex education is not like education of history or geography. We have sexual organs and with getting information, it is possible that we desire to stimulate ourselves...' (FGD2).

Also, some participants believed that providing primary information about sexuality will stimulate individuals' curiosity to search for more sexuality information. Soraya (27 years) stated that:

'When a person is prone to initiate her sexual relationships, receiving sexual information will make her thirsty for more information and motivate her for initiating sexual relationship' (FGD1).

These young women believed that they might initiate their sexual relationships if they receive sex education because they view sex education as a motivation for sexual awareness.

\subsubsection{Tainted Modesty}

The participants described sexual modesty as the inclusion of ethical and religious modalities in sexual behaviors. Several participants stated that sex education could destroy modesty. Nazila, 23 years old, is one of the participants who did not agree with sex education: 
'Providing sex education looks like giving out a license for immoral sexual behaviors; in fact, if we receive sex education, it is possible that we think premarital sexual behaviors are ethical phenomena and then haya (modesty) boundaries will be destroyed' (FGD3).

Anis, 19 years old, stated that:

'....you know, sexual modesty in Iran is completely related to premarital sexual abstinence, especially for women. People think if sex education is provided, sexual chaos will happen and people will not able to control themselves. Although, it could not be true for all people and all societies, what is obvious is that people feel more freedom and it could destroy sexual abstinence and modesty' (ind.inw).

Some participants believed that one of bad effects of sex education could be tainting modesty, which may provide a justification for premarital sexual behaviors. They thought sex education might provide a license for avoiding abstinence and destroying modesty.

\section{Discussion}

The findings indicated that women believed sex education could have three important advantages: increased knowledge, normalization of sexuality issues, and improved self-efficacy. We found that most participants did not receive school-based sex education, although they obtained sexual information from different sources. Similarly, a study from Iran showed that the source of sexuality information for adolescents were close friends, magazines, books, media, health-care professionals, religious figures, and families (4). Lack of sex education might be due to the fact that in a number of countries such as Iran, providing sex education for young people poses difficulties. In contrast, a number of countries, such as Pakistan and India, have certain strategy and curriculum-based sex education programs for adolescents and youth (17).

The process of normalization of sexual and reproductive issues that our participants described seems to be similar to the UNESCO's forefront agenda, which states that the normalization of sexual issues is an important means to change social norms, facilitate communication about sexuality, remove social and attitudinal obstacles, and increase knowledge (18). In the Iranian tradition, sexuality is a sensitive topic and thus, some people may believe that young unmarried individuals do not need sex education before marriage (4). Perhaps in such situations there is the need to reassure people that the goal of sex education is not to change cultural values and that it could help young people, and the society as a whole, to benefit from preventing some drawbacks, such as tainting modesty or initiating premarital sexual relationships.
Sex education helps young people make appropriate decisions in critical situations. As one of important concepts of social cognitive theory, self-efficacy, as a personal sense of control and action to solve problems, would make people feel more committed to their decisions (19). BayCheng is argued that improved self-efficacy in young people should be considered as a key feature of a useful sex education program (20). Some scholars refer to this skill as sexual self-efficacy and break it down into 3 main parts: the capability to reject unintended sexual activities, to assert sexual desires, and to be cautious in sexual behaviors (21). Our participants mainly focused on 2 aspects of sexual self-efficacy: the capability to reject unintended sexual activities and to be cautious in sexual behaviors.

Additionally, participants perceived 2 disadvantages for sex education: motive for initiating sexual behavior and tainted modesty. Adults may think adolescents will try sex if they receive sex education because they may view sex education as a motivation for sexual awareness. There are 2 perspectives to address this matter: a contextual and religious perspective and a scientific point of view. Although the population of Iran has a high proportion of Muslims and abstinence is the assumed behavior for unmarried people (22), it seems the main reason Muslim people may be opposed to sex education is due to the cultural nurture, not the religious beliefs. In the Quran, God cares for all aspects of human life and discusses reproduction, creation, family life, menstruation, and even ejaculation. It is therefore quite possible and appropriate for sex education to be provided in Muslim societies according to Islamic ideology (4). From the scientific perspective, multiple studies have showed that comprehensive sex education could not be sexually erotic if it is age-appropriate and is provided accurately (23).

A few of our participants thought sex education provides a context in which a license is given for premarital sexual relationships. Modesty holds a particularly important meaning in Islamic societies such as Iran. A qualitative investigation on Iranian women reported that participants linked the language of modesty to the lack of sexual self-efficacy, powerlessness in decision-making, and increasing sexual health problems, due to the fact that they did not feel free to talk about sex openly (22).

Although the legitimacy and content of sex education in school has frequently been criticized (21), studies suggest that receiving sex education before the first sexual experience is associated with avoiding early sexual relationships, using more contraception, and preventing sexually transmitted infections $(7,13)$. Although existing studies focus on non-Iranian contexts, the present study suggest that young women in the Iranian culture confirmed that sex education in this culture could provide the knowledge and 
skills to make healthy and informed decisions regarding their sexual relationships.

\subsection{Conclusion}

The findings indicated that sex education might have 3 important advantages for young women: increase their knowledge, help to talk openly on sexuality issues, and improve their self-efficacy. Disadvantages of sex education in the viewpoints of participants could be a motive for initiating sexual behavior and tainted modesty. Findings of the study could be used to design a culturally appropriate sex education in conservative contexts, such as Iran.

\section{Acknowledgments}

This study was originated from the first investigator's thesis for receiving a PhD degree in reproductive health, at faculty of medical sciences, Tarbiat Modares University, Tehran, Iran. We would like to thank all the young women who participated in this study.

\section{References}

1. Ramiro L, Reis M, Matos MG, Diniz JA. Sex education among portuguese adolescent students. Procedia Soc Behav Sci. 2011;29:493-502. doi: 10.1016/j.sbspro.2011.11.268.

2. UNFPA.Population and reproductive health indicators. New York, United States: United Nations Population Fundm, (UNFPA); 2011.

3. Holzner BM, Oetomo D. Youth, sexuality and sex education messages in Indonesia: issues of desire and control. Reprod Health Matters. 2004;12(23):40-9. [PubMed: 15242209].

4. Farahani FK, Cleland J, Mehryar AH. Associations between family factors and premarital heterosexual relationships among female college students in Tehran. Int Perspect Sex Reprod Health. 2011;37(1):30-9. doi: 10.1363/3703011. [PubMed: 21478086].

5. Zangeneh N. A critical view of sexual health education in iran. India: In Plainspeak, a digital magazine on sexuality in the Global South; 2013. Available from: http://www.tarshi.net/inplainspeak/a-critical-viewof-sexual-health-education-in-iran/.

6. IranSCo . The overall results of general population and housing census. Tehran: Statistical Centre of Iran; 2011.

7. Kirby DB, Laris BA, Rolleri LA. Sex and HIV education programs: their impact on sexual behaviors of young people throughout the world. J Adolesc Health. 2007;40(3):206-17. doi: 10.1016/j.jadohealth.2006.11.143. [PubMed:17321420].

8. Mohamadi M. Knowledge, attitude and behavior of adolescent boys aged 15 to 18 in Tehran on reproductive health. Fertil Infertil J.2002:23750 .
9. Nasirian M, Doroudi F, Gooya MM, Sedaghat A, Haghdoost AA. Modeling of human immunodeficiency virus modes of transmission in Iran.J Res Health Sci. 2012;12(2):81-7. [PubMed: 23241516].

10. Qaderi S. The third wave of transmission of HIV is increasing. Tehran: khabaronline News Agancy; 2010. Available from: http://www. Khabaronline.ir/news-85500aspx.

11. Tavoosi A, Zaferani A, Enzevaei A, Tajik P, Ahmadinezhad Z. Knowledge and attitude towards HIV/AIDS among Iranian students. BMC Public Health. 2004;4:17. doi: 10.1186/1471-2458-4-17. [PubMed: 15157281].

12. Ancheta R, Hynes C, Shrier LA. Reproductive health education and sexual risk among high-risk female adolescents and young adults. J Pediatr Adolesc Gynecol. 2005;18(2):105-11. doi: 10.1016/j.jpag.2005.01.005. [PubMed: 15897107].

13. Mueller TE, Gavin LE, Kulkarni A. The association between sex education and youth's engagement in sexual intercourse, age at first intercourse, and birth control use at first sex. J Adolesc Health. 2008;42(1):89-96. doi: 10.1016/j.jadohealth.2007.08.002. [PubMed: 18155035].

14. Hsieh HF, Shannon SE. Three approaches to qualitative content analysis. Qual Health Res. 2005;15(9):1277-88. doi: 10.1177/1049732305276687. [PubMed: 16204405].

15. Merghati Khoei E, Whelan A, Cohen J. Sharing beliefs: what sexuality means to Muslim Iranian women living in Australia. Cult Health Sex. 2008;10(3):237-48. doi: 10.1080/13691050701740039. [PubMed: 18432423].

16. Streubert HJ, Carpenter DR. Qualitative research in nursing, advancing the humanistic imperative. United States: Lippincott Williams and Wilkins; 2010.

17. UNESCO. Review ofpolicies and strategies to implement and scale up sexuality education in Asia and the Pacific. Thailand: UNESCO Bangkok; 2012.

18. UNESCO. International guidelines on sexuality education, an evidence informed approach to effective sex, relationships and HIV/STI education. United Kingdom: UN Educational, Scientific and Cultural Organisation, (UNESCO); 2009. Available from: http://www.refworld.org/ docid/4a69b8902.html.

19. Schwarzer R, Fuchs R. Self efficacy and health behaviours. Predicting health behaviour, research and practice with social cognition models. United Kingdom: Open University Press;1996. p. 163-96.

20. Bay Cheng LY. The trouble of teen sex, the construction of adolescent sexuality through school based sexuality education. Sex Educ. 2003;3(1):61-74. doi: 10.1080/1468181032000052162.

21. Illes J. Young sexual citizens, reimagining sex education as an essential form of civic engagement. Sex Educ. 2012;12(5):613-25. doi: 10.1080/14681811.2011.634152.

22. Merghati Khoei E. Language of love in culture of silence, Iranian womens sexual understandings and sociocultural. Australia: School of Public Health and Community Medicine, Faculty of Medicine, University of New South Wales; 2006.

23. Eisenberg ME, Bernat DH, Bearinger LH, Resnick MD. Support for comprehensive sexuality education: perspectives from parents of school-age youth. J Adolesc Health. 2008;42(4):352-9. doi: 10.1016/j.jadohealth.2007.09.019. [PubMed: 18346660]. 